Documentation et bibliothèques

DOCUMENTATION BIBLIOTHEQUES

\title{
Les bibliothèques universitaires canadiennes, dix ans après...
}

\section{Daniel Reicher}

Volume 21, numéro 1, mars 1975

URI : https://id.erudit.org/iderudit/1055517ar

DOI : https://doi.org/10.7202/1055517ar

Aller au sommaire du numéro

\section{Éditeur(s)}

Association pour l'avancement des sciences et des techniques de la documentation (ASTED)

\section{ISSN}

0315-2340 (imprimé)

2291-8949 (numérique)

Découvrir la revue

Citer cet article

Reicher, D. (1975). Les bibliothèques universitaires canadiennes, dix ans après... Documentation et bibliothèques, 21(1), 37-40.

https://doi.org/10.7202/1055517ar

\section{Résumé de l'article}

En décembre 1964, C.A.C.U.L. recommandait à la Commission Bladen que le budget de la bibliothèque universitaire représente dix pour cent du budget de fonctionnement de l'université. Dix ans après, on peut constater que cette recommandation est restée lettre morte tant au Canada qu'au Québec.
Tous droits réservés (C) Association pour l'avancement des sciences et des techniques de la documentation (ASTED), 1975
Ce document est protégé par la loi sur le droit d'auteur. L'utilisation des services d'Érudit (y compris la reproduction) est assujettie à sa politique d'utilisation que vous pouvez consulter en ligne.

https://apropos.erudit.org/fr/usagers/politique-dutilisation/ 


\title{
Les bibliothèques universitaires canadiennes, dix ans après...
}

\author{
Danlel Relcher \\ Professeur agrégé \\ École de bibliothéconomie \\ Université de Montréal
}

En décembre 1964, C.A.C.U.L. recommandait à la Commission Bladen que le budget de la bibliothèque universitaire représente dix pour cent du budget de fonctionnement de l'université. Dix ans après, on peut constater que cette recommandation est restée lettre morte tant au Canada qu'au Québec.

In December 1964, C.A.C.U.L. recommended to the Bladen Commission that the budget of the university libraries should represent ten per cent of the university operating budget. Ten years later, it is apparent that the recommendation has never been brought into operation either in Canada or in Quebec.

En diciembre 1964, C.A.C.U.L. recomendaba a la Comisión Bladen que el presupuesto de la biblioteca universitaria representara el diez por ciento del presupuesto de funcionamiento de la universidad. Diez años más tarde, se puede observar que esta recomendación se ha quedado sin cumplir tanto en el Canadá como en la provincia de Quebec.

En décembre 1964, le Canadian Association of College and University Libraries (C.A.C.U.L.) présentait son Forecast of the cost of academic library services in Canada, 1965-1975' à la Commission faisant enquête sur le financement de l'enseignement supérieur, connue familièrement sous le nom de son président, le professeur Bladen. Le climat universitaire était à l'euphorie hyperbolique.

Revenues du choc du spoutnik, les universités étaient embrayées à la vitesse de rattrapage, au point que les eggheads étaient même devenus respectables en Amérique du Nord. Naturellement, les bibliothèques universitaires avaient avancé d'abord un orteil prudent, avant d'emboîter le même pas guilleret, mais elles étaient arrivées à la bonne cadence avec un décalage d'à peine quelques années. En novembre 1961, C.A.C.U.L. se mettait à rédiger ses premières normes et allait informer la communauté universitaire en 1963 que soixante-quinze volumes étaient nécessaires par

1. Association canadienne des bibliothèques de collèges et d'universités, Forecast of the Cost of Academic Library Services in Canada, 1965-1975, Waterloo, Ont., University of Waterloo Press, 1964 étudiant. ${ }^{2}$ En 1962 , le rapport Williams ${ }^{3}$ confirmait qu'il y avait du chemin à faire dans les bibliothèques universitaires canadiennes, pour les sciences humaines et sociales. Si nous nous faisions, encore une fois, secouer les puces en 1964, lorsque Béatrice Simon ${ }^{4}$ évalua nos bibliothèques médicales, nous arrivions quand même en ville. Finis les complexes d'infériorité: toute université qui se respecte devait avoir son million de volumes.

En décembre 1964, donc, nous nous étions tellement bien identifés aux objectifs de l'enseignement supérieur que nous réclamions hardiment notre dîme. Le mémoire de C.A.C.U.L. fut entériné par la Commission Bladen et sa recommandation principale, de

2. Association canadienne des bibliothèques de collèges et d'universités, Guide to Canadian University Library Standards, Ottawa, 1965, p. 19

3. Edwin E. Williams, Resources of Canadian University Libraries for Research in the Humanities and Social Sciences, Ottawa, National Conference of Canadian Universities and Colleges, 1962.

4. Beatrice V. Simon, Library Support of Medical Education and Research in Canada, Ottawa, Association of Canadian Medical Colleges, 1964. 
réserver dix pour cent du budget de fonctionnement de l'université à la bibliothèque, était inscrite pour jamais dans notre catéchisme bibliothéconomique, sur la page où l'on fait alIusion au Cœur de I'Université. Sur quoi se basait cette demande? Tout simplement sur le fait qu'en 1962/1963, les bibliothèques universitaires recevaient environ $4.7 \%$ des budgets universitaires de fonctionnement. Si Williams et Simon avaient raison de souligner nos lacunes, C.A.C.U.L. espérait qu'en doublant les budgets, on pourrait faire du rattrapage.

En 1967, le rapport Downs sur les Ressources des bibliothèques d'université et de recherche au Canada ${ }^{5}$ venait étayer nos exigences. Étant donné que ce rapport était publié par l'Association des Universités et Collèges du Canada, nous pouvions prendre pour acquis que la communauté universitaire nous appuyait. La bataille des bibliothèques était gagnée!? D'ailleurs, soucieux de ne plus nous faire accuser de myopie, nous nous arrangions pour faire ouvrir des écoles de bibliothéconomie à gauche (Alberta), à droite (Dalhousie) et au centre (Western Ontario).

En 1969, les ministères de l'Éducation ne pouvaient créer les universités assez instantanément à leur gré, au point que l'Annuaire du Canada fournissait des statistiques pour 73 bibliothèques universitaires en $1969 / 70$ et pour 108 en 1970/71. Selon les us et coutumes du milieu, la bibliothèque suivait toujours l'université, mais elle était parfois entourée de bien peu de tendresse. Vous vous souviendrez sans doute de la déclaration fracassante d'un recteur du temps, qui peut se résumer en une petite phrase sans nuance: là où il y a assez de livres pour trois universités, il $y$ en a assez pour une quatrième.

En septembre 1969, C.A.C.U.L. décidait que ses normes de 1961 étaient dépassées, et en mai 1971, un projet de normes révisées était déposé. 6 Les exigences budgétaires étaient codifiées, explicitées, devenaient quantifiables... L'euphorie se tenait encore sur la branche positive de l'hyperbole, au point que les membres de C.A.C.U.L. trouvèrent que le projet était trop étriqué pour décrire l'avenir glorieux des bibliothèques universitaires.

5. Robert B. Downs, Resources of Canadian Academic and Research Libraries, Ottawa, Association of Universities and Colleges of Canada, 1967.

6. Association canadienne des bibliothèques de collèges et d'universités, Trends for the Seventies; Guidelines for Canadian University Libraries, Montréal, 1971.
Pourtant l'élan perdait un peu de son élasticité, sans doute à cause des lois de la pesanteur... administrative, s'entend. Quelques oiseaux de mauvais augure venaient tacheter notre beau panorama bibliothéconomique. En effet, certains diplômés universitaires faisaient le pied de grue devant les bureaux de recrutement. Les employeurs ne se dérangeaient plus pour venir embaucher les diplômés en bibliothéconomie en janvier; ils attendaient bien tranquillement chez eux jusqu'en mai.

En 1973, l'hyperbole semblait s'orienter vers la négative. Pour la première fois au $\mathrm{Ca}$ nada, une université décidait de fermer son école de bibliothéconomie. Le réveil était rude. Le pire s'en venait: la stabilisation budgétaire en période d'inflation galopante. Aux États-Unis, la situation était tout aussi sombre. En mars 1973, Downs et Mc Anally 7 demandaient à leurs collègues directeurs de bibliothèques universitaires ce qu'ils pensaient de ce début de sclérose, et ceux-ci répondaient en 1974 qu'il s'agissait de lassitude sénile. ${ }^{8}$

Avant de sombrer dans le masochisme, les bibliothécaires se doivent d'examiner la situation avec lucidité. Ce réveil en sursaut était-il aussi imprévisible qu'on le prétend? Notre daltonisme nous aurait-il fait confondre le graphique vert espérance avec le graphique rouge catastrophe?

En 1965, le président Spinks se demandait, à l'université de la Saskatchewan, si les bibliothèques universitaires pouvaient encore se développer sans frein. En 1966, il se demandait si même l'Ontario pouvait se payer le luxe de l'autonomie universitaire absolue. ${ }^{9}$

En 1967, la Direction générale de l'enseignement supérieur du Québec se penchait sur des normes de financement pour les bibliothèques universitaires. En 1970, le Conseil des universités du Québec déclarait ${ }^{10}$ que les

7. Robert B. Downs, "Changing roles of directors of university libraries", College and Research Libraries, vol. 34 (1973), 103-125.

8. Association of College and Research Libraries, Seminar on the Changing Role of Directors of University Libraries, Chicago, 1974. (cassette)

9. Commission to Study the Development of Graduate Programmes in Ontario Universities, Report to the Committee on University Affairs and the Committee of Presidents of Provincially-Assisted Universities (J.W.T. Spinks, chairman), Toronto, 1966.

10. Conseil des universités, Deuxième rapport annuel, 1970-1971, Québec, 1971, p. 31. 
bibliothèques universitaires québécoises devaient s'automatiser avant d'obtenir des budgets plus considérables. Cette déclaration s'orientait bien dans une ligne de pensée futuriste, mais un peu de réalisme administratif aurait fait percevoir aux bibliothécaires certaines assonances sinistres. Si l'on automatise une bibliothèque, c'est, paraît-il, pour contenir les coûts de fonctionnement. Le Conseil des universités recommandait donc de stabiliser les budgets de bibliothèques, qu'elles s'automatisent ou pas.

En 1973, nos collègues pèlerins allaient admirer la bibliothèque de l'université de Toronto et se demandaient, envieusement ou mélancoliquement, si c'était, pour longtemps, le dernier temple érigé à la documentation.

Mais, au fait, l'optimisme des bibliothécaires avait-il jamais été justifié? Si l'on avait examiné de plus près les statistiques, n'aurait-on pas pu vérifier si la norme de dix pour cent avait déjà été atteinte ou même approchée?

Nous nous proposons de répondre à cette question. La méthodologie utilisée est élémentaire: comparer le budget universitaire de fonctionnement avec celui de la bibliothèque, multiplié par dix. Les informations statistiques sont extraites surtout de l'Annuaire du Canada et accessoirement de celui du Québec.
A l'examen des statistiques compilées dans les Annuaires, l'on se pose naturellement des questions sur la nature des activités mesurées. Les dépenses de fonctionnement incluent-elles celles de la recherche? Les dépenses universitaires incluent-elles les dépenses d'investissement? Comment se fait-il que les montants, pour une même année, puissent changer d'une édition à une autre de l'Annuaire? Les dépenses de bibliothèque couvrent-elles les mêmes postes budgétaires pour toutes les universités? Le graphique qui suit souligne l'illusion de vouloir interpréter trop strictement le fatras chiffré que l'on nous offre comme description statistique de la situation. Pourtant une image se dégage à grands traits.

Les courbes $A$ et $B$ représentent les dépenses universitaires globales avec un changement de méthodologie en 1969. La courbe $A^{\prime}$ est une extrapolation basée sur l'Annuaire $d u$ Québec. Les courbes $C$ et $D$ représentent les dépenses des bibliothèques universitaires multipliées par dix avec un changement de méthodologie en 1970. La courbe C continue l'ancienne pratique d'additionner ensemble les bibliothèques de collèges et d'universités. La courbe $D$ exclut les bibliothèques de collèges. La courbe $E$ multiplie les dépenses des bibliothèques universitaires par VINGT.

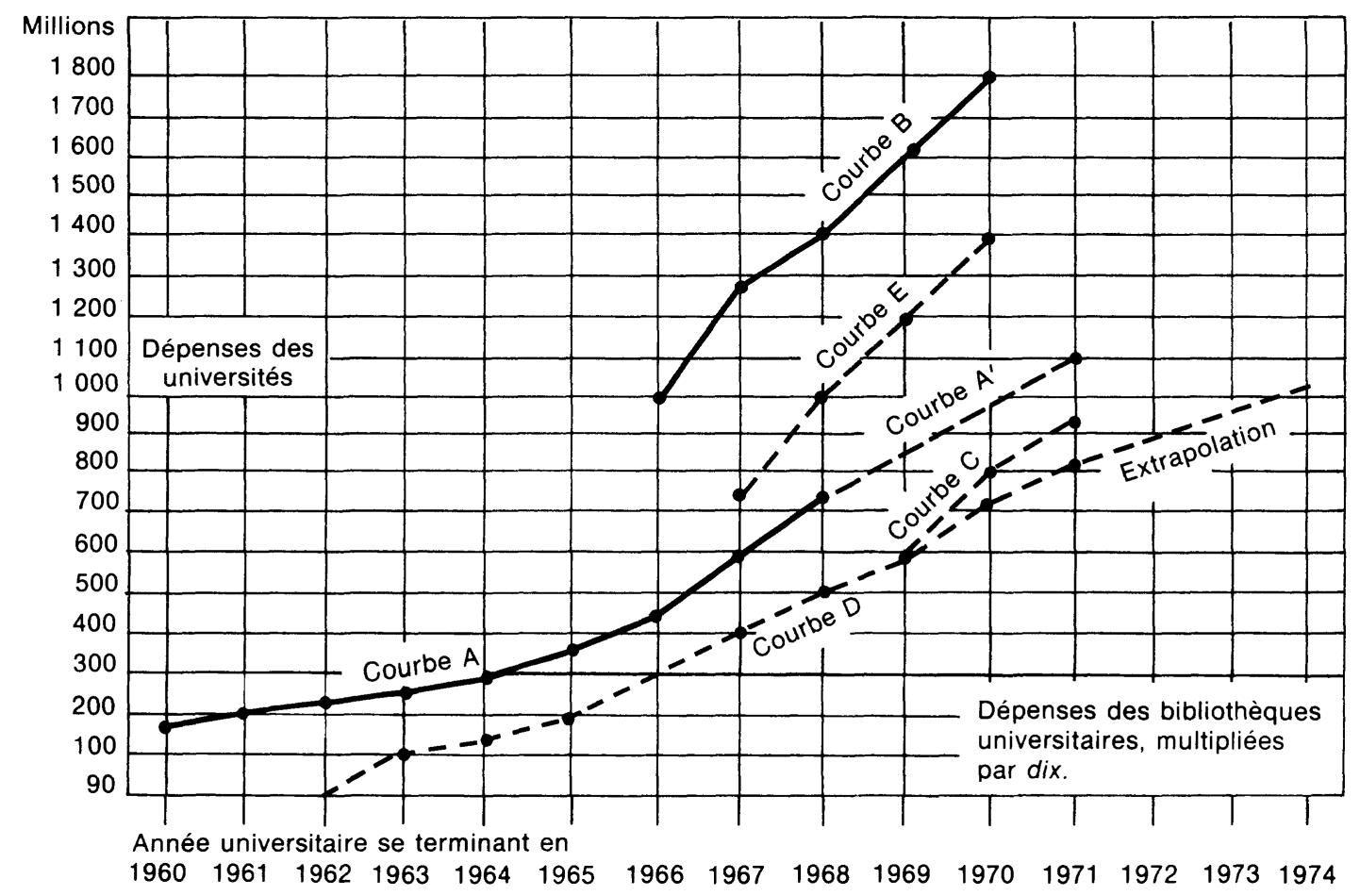


Les conclusions à tirer du graphique sont claires:

$1^{\circ}$ la norme de dix pour cent n'a jamais été atteinte par l'ensemble des bibliothèques universitaires canadiennes;

$2^{\circ}$ l'écart entre les courbes $A$ et $C$ ne manifeste aucune tendance à se rétrécir, et indique que les budgets de bibliothèque ont deux années de retard sur les budgets universitaires;

$3^{\circ}$ l'écart entre les courbes $B$ et $D$ est prodigieux. Seule la courbe E, qui multiplie les dépenses des bibliothèques universitaires par VINGT (5\%) au lieu de DIX (10\%), est plus ou moins parallèle à la courbe $B$.

Statistique-Canada n'a pas encore publié de chiffres pour la période 1971-1974 et il n'est donc pas possible de continuer le graphique. Cependant, le lecteur voudra bien se souvenir du fait que la période 1967-1971 en était une d'euphorie hyperbolique.

Peut-on accuser C.A.C.U.L. d'avoir été mauvais prophète en décembre 1964? Pas du tout. Une de ses deux hypothèses de développement prévoyait, pour 1974, des dépenses de l'ordre de $\$ 66,000,000$, en dollars de 1963 , ce qui donnerait à peu près $\$ 110,000,000$, en dollars de 1974, à cause de l'inflation d'environ $70 \%$. Une extrapolation rectiligne de la courbe $D$ arriverait assez près de ce montant.

Ce que C.A.C.U.L. ne pouvait pas accepter comme hypothèse de travail, c'était la croissance des universités plus rapide que celle des bibliothèques, et pourtant c'est justement là le décalage qui s'est produit. On touche du doigt le drame des bibliothèques: il n'y a jamais eu de rattrapage durant la décennie 1964-1974; il n'y a eu qu'une vaine poursuite.

Si l'on examine la situation au Québec, on remarque un état de fait encore plus déprimant qu'ailleurs. En 1968/69, nous dépensions $\$ 11,029,000$ pour nos bibliothèques universitaires, alors que la norme de $10 \%$ nous aurait donné ce budget en 1964/65. En $1970 / 71$, nous dépensions $\$ 14,323,000$ alors que nous aurions dû le faire en 1966/67.

Pendant combien de temps encore faudra$t$-il que les chercheurs québécois acceptent d'utiliser des bibliothèques dont les ressources traînent quatre ans en arrière par rapport à celles fournies à l'enseignement, alors que les chercheurs canadiens se plaignent déjà de devoir subir les conséquences d'un décalage budgétaire qui n'est que de deux années?

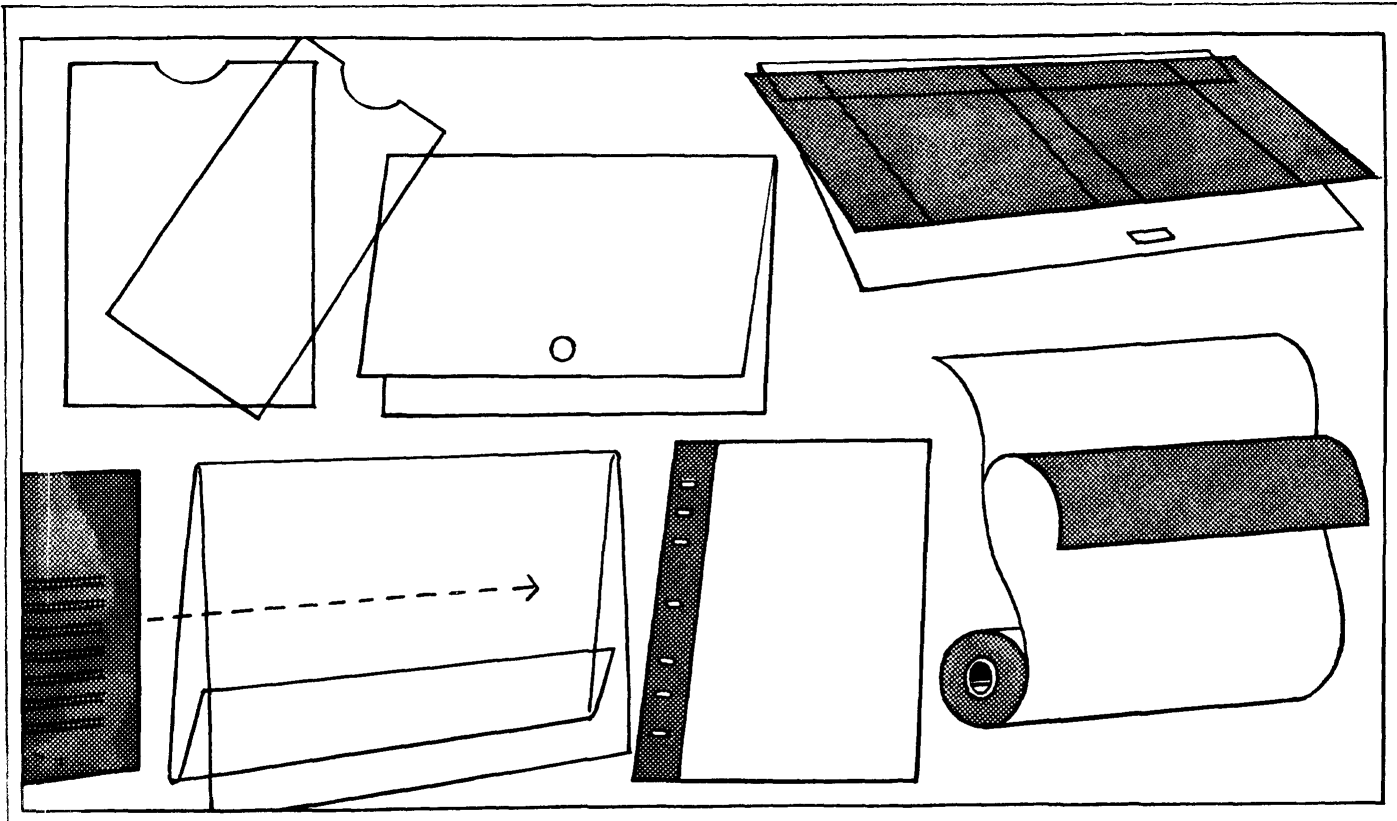

PROTECTION PLASTIQUE - PROTĖGE-DOCUMENTS - MATÉRIEL DE RÉPARATIONS

R.M.

LEDUC

\& CIE

DEMANDEZ NOTRE CATALOGUE

2101, RUE BENNETT - MONTRÉAL H1V 2T4 - TÉL.: 255-1221 